PTU-160 SUCCESSFUL CLEARANCE OF CHRONIC NOROVIRAL INFECTION BY RIBAVIRIN IN A PATIENT WITH COMMON VARIABLE IMMUNODEFICIENCY-ASSOCIATED ENTEROPATHY RESULTS IN COMPLETE SYMPTOMATIC AND HISTOPATHOLOGICAL RESOLUTION

doi:10.1136/gutjnl-2012-302514c.160

${ }^{1} \mathrm{~J}$ Woodward, ${ }^{* 2} \mathrm{~A} \mathrm{Ng},{ }^{3} \mathrm{~A}$ Aravinthan, ${ }^{4} \mathrm{~B}$ Bandoh, ${ }^{4} \mathrm{H}$ Liu, ${ }^{4} \mathrm{~S}$ Davies, ${ }^{5} \mathrm{P}$ Stevenson, ${ }^{2} \mathrm{M}$ Curran, ${ }^{5} \mathrm{D}$ Kumararatne. ${ }^{1}$ Gastroenterology, Clinical Microbiology and Public Health Laboratory, Cambridge, UK; ${ }^{2}$ Health Protection Agency, Clinical Microbiology and Public Health Laboratory, Cambridge, UK; ${ }^{3}$ Hepatology, Addenbrookes Hospital, Cambridge, UK; ${ }^{4}$ Histopathology, Addenbrookes Hospital, Cambridge, UK; ${ }^{5}$ Clinical Biochemistry and Immunology, Addenbrookes Hospital, Cambridge, UK

Introduction We have recently demonstrated an association between chronic Noroviral infection and Common Variable Immunodeficiency-associated (CVID) enteropathy. Here we describe a patient with CVID enteropathy treated with Ribavirin.

Methods A 33-year-old lady with known CVID presented to our service in 2003 with 20\% weight loss, nausea and profuse diarrhoea. Investigations revealed classical appearances of CVID enteropathy with subtotal duodenal villous atrophy, but no evidence of bacterial, enteroviral or parasitic infection. Treatment with gluten withdrawal, elemental diet, and budesonide were largely ineffective and she required parenteral nutrition for malabsorption and anti-TNF $\alpha$ therapy with Infliximab and subsequently Humira for symptom relief. No therapy changed the degree of villous atrophy. Following 6 years of symptoms, stool was noted to be positive on PCR for Norovirus RNA. Retrospective analysis of archived duodenal biopsies revealed the presence of Noroviral RNA in all biopsies from 2003 to 2009 and RNA sequencing showed this to be the same strain of virus throughout. In view of reported in vitro activity of Ribavirin against Norovirus, this agent was commenced with therapeutic level monitoring and quantitative stool PCR for Norovirus excretion.

Results Once Ribavirin levels were $>1000 \mathrm{ng} / \mathrm{ml}$, quantitative PCR demonstrated a reduction in Noroviral excretion which then became undetectable. Simultaneously, the patient reported dramatic symptomatic relief with a change from profuse diarrhoea to two formed motions a day, no nausea and return of appetite. Parenteral nutrition and Humira were stopped. Duodenal biopsies after 3 months showed complete resolution of villous morphology and were negative for Norovirus on PCR. Stool remained negative for Noroviral RNA. After 6 months Ribavirin therapy was stopped. The patient has remained asymptomatic for the subsequent 9 months with no evidence of recurrent Noroviral excretion.

Conclusion Complete resolution of symptoms and duodenal villous atrophy with clearance of the Norovirus suggests that the association of Norovirus infection with CVID enteropathy is causal. Ribavirin may have activity against Norovirus in vivo and this first ever demonstration of a cure for this condition requires confirmation in other cases.

Competing interests None declared.

\section{PTU-161 CHRONIC NOROVIRUS INFECTION AS A CAUSE OF COMMON VARIABLE IMMUNODEFICIENCY-ASSOCIATED ENTEROPATHY}

doi:10.1136/gutjnl-2012-302514c.161

\begin{abstract}
${ }^{1} \mathrm{~J}$ Woodward, ${ }^{* 2} \mathrm{~A} \mathrm{Ng},{ }^{3} \mathrm{~A}$ Aravinthan, ${ }^{4} \mathrm{~B}$ Bandoh, ${ }^{4} \mathrm{H}$ Liu, ${ }^{4} \mathrm{~S}$ Davies, ${ }^{5} \mathrm{P}$ Stevenson, ${ }^{2} \mathrm{M}$ Curran, ${ }^{5} \mathrm{D}$ Kumararatne. ${ }^{1}$ Gastroenterology, Clinical Microbiology and Public Health Laboratory, Cambridge, UK; ${ }^{2}$ Health Protection Agency, Clinical Microbiology and Public Health Laboratory, Cambridge, UK; ${ }^{3}$ Hepatology, Addenbrookes Hospital, Cambridge, UK; ${ }^{4}$ Histopathology, Addenbrookes Hospital, Cambridge, UK; ${ }^{5}$ Clinical Biochemistry and Immunology, Addenbrookes Hospital, Cambridge, UK
\end{abstract}

Introduction A severe enteropathy with villous atrophy occurs in up to $15 \%$ of patients with common variable immunodeficiency
(CVID). This study set out to determine the role of Norovirus infection in this condition.

Methods Stool and archived small intestinal biopsies from patients with CIVD enteropathy were analysed by PCR for the presence of Norovirus RNA. PCR products were sequenced to determine the relationship of viral isolates. Stool samples from ten asymptomatic patients with CVID served as controls.

Results All seven patients in our CVID cohort with enteropathy and villous atrophy showed persistent faecal norovirus excretion. No other entero- or parechovirus infection was apparent in any patient. Analysis of archived duodenal biopsies demonstrated the presence of the same viral strain (genotype II.4) in individuals over periods of up to 8 years. The presence of norovirus was strongly associated with villous atrophy in all our cases. Asymptomatic CVID patients showed no evidence of norovirus excretion.

Conclusion Chronic norovirus infection occurs in patients with common variable immunodeficiency-associated enteropathy and is strongly associated with villous atrophy and symptomatic malabsorption in all patients with CVID in this cohort. Chronic Norovirus is implicated as a major cause of CVID enteropathy.

Competing interests None declared.

\section{PTU-162 CAPSULE ENDOSCOPY: DIAGNOSTIC YIELD AND CLINICAL OUTCOMES IN NORTH-EAST LINCOLNSHIRE}

doi:10.1136/gutjnl-2012-302514c.162

J R White, ${ }^{*}$ R Mudawi, A Naqvi, S Enaganti. Gastroenterology, Diana, Princess of Wales Hospital, Grimsby, UK

Introduction Capsule Endoscopy (CE) has become a very important non-invasive tool for the investigation of small bowel pathology. This study examines the patient pathway to CE, from initial referral to final clinical outcome, within our newly established service.

Methods Medical notes for patients who underwent CE in North East Lincolnshire from 2009 to 2011 were reviewed retrospectively. Information analysed included demographics, previous investigation, diagnosis and subsequent treatment

Results 123 patients underwent CE in the 2-year study period. 22 notes were unobtainable, so 101 were analysed. The study population characteristics include a median age of 59 (16-76 yrs), an equal gender spread ( $53 \%$ female and $47 \%$ male) with a $99 \%$ white ethnic majority. $95 \%$ of referrals were as an outpatient and $92 \%$ were made by physicians. The median waiting time was 115 days. The main indications were: recurrent iron deficiency anaemia (48.5\%), inflammatory bowel disease (IBD) (33.7\%), overt gastrointestinal (GI) bleeding (15\%). Patients had commonly undergone previous investigations: marjority had gastroscopy, colonoscopy (82.3\%), CT abdomen (22.8\%), Barium meal (21.8\%), small bowel MRI (18.8\%) and flexi-sigmoidoscopy (9.9\%). 6.9\% (7 patients) had undergone a previous CE. CE detected abnormality in $97 \%$ of this cohort, although some were insignificant. The most common pathology was: erosions (54.5\%), evidence of blood (41.6\%), angiodysplastic lesions (32.6\%), ulceration (28.7\%), focal inflammation (26.7\%) and upper GI inflammation (19.8\%). $72.2 \%$ of pathology was in the small bowel. Procedural complications included capsule retention (2), and suboptimal views in four patients. CE reporting confirmed diagnoses of angiodysplasia (25.7\%), IBD (16.8\%), drug induced small bowel erosions (6.9\%), duodenitis/gastritis/ oesophagitis $(5.9 \%)$ and ulceration $(4.9 \%)$. Rarer diagnoses included small bowel tumour and Meckel's Diverticulum. Median reporting time was 35 days. CE significantly contributed to the management of the patients examined: $78 \%$ had changes to their medications (eg, stop NSAIDs, start IBD drugs), $62 \%$ underwent further 
investigations (eg, enteroscopy, gastroscopy and colonoscopy), 52\% underwent therapeutic intervention (eg, APC, polypectomy and surgical referral). However, in $30 \%$ their management was unchanged. The majority of patients (88.1\%) are still under active follow-up today.

Conclusion The CE experience at this centre is of a simple, welltolerated investigation which allows definite diagnoses to be made with minimal complications. Higher diagnostic yield compared to previous published data could be explained by the strict inclusion criteria and recent introduction of this new service. This expanding service highlights the need for more resources to reduce waiting and reporting times in line with other GI investigations.

Competing interests None declared.

\section{PTU-163 DEPRESSION AND CARCINOID SYNDROME: IS THERE ANY RELATIONSHIP? A CROSS-SECTIONAL STUDY}

doi:10.1136/gutjnl-2012-302514c.163

1J Garcia-Hernandez, ${ }^{1} \mathrm{M}$ Mohmaduvesh, ${ }^{1} \mathrm{P}$ Davies, ${ }^{1} \mathrm{C}$ Toumpanakis, IJ R Goodhand, 'M Caplin, ${ }^{2} \mathrm{D}$ Skuse. ${ }^{1}$ Royal Free Hospital, London, UK; ${ }^{2}$ UCLH, London, UK

Introduction The relationship between brain serotonin and depression is well established. It is also widely accepted that serotonin hyperproduction in carcinoid syndrome does not cross the brain barrier. CNS serotonin is synthesised from tryptophan within serotonergic neurons and a deficiency of this precursor could be possible on carcinoid patients. In this cross-sectional study we evaluated, whether the feelings of depression are associated with neuroendocrine symptomatology. In addition, whether self-report of quality of life is associated with feelings of depression.

Methods 47 patients with carcinoid syndrome completed a survey via clinic involving: QoL questionnaires and the Beck's Depression Inventory-II (BDI-II). All questionnaires were counterbalanced.

Results 45 out of 47 patients had low scores on the BDI-II and not likely to suffer from depression. Only two participants had moderate depression scores. These scores were negatively associated with self-report of QoL, $r=-0.59, \mathrm{~N}=46, \mathrm{p}<0.001$. Interestingly, endocrine symptoms that is, flushing and night sweats, were not associated with depressive symptoms: $\mathrm{r}=0.22, \mathrm{~N}=44, \mathrm{p}=0.15$, whereas, gastrointestinal symptoms that is, diarrhoea, abdominal discomfort, bloatedness and indigestion, were strongly associated with depression scores: $r=56, N=43, p<0.001$.

Conclusion Depressive scores in carcinoid patients are often attributed to the stress of diagnoses and adaptation to this chronic disease, however, although a causal relationship has not been established between carcinoid and depression, physicians should consider this possibility when psychological symptoms do not improve with conventional therapy. Further research is needed to understand why gastrointestinal, and not endocrine, symptoms were correlated with depressive symptoms.

Competing interests None declared.

\section{REFERENCES}

1. Lapeire LD, Tansens A, Lemmens G, et al. Carcinoid encephalopathy: A single entity or a spectrum of different disorders. Acta Oncologica 2010;49:268-70

2. Major LF, LaVonne Brown G, Wilson WP. Carcinoid and psychiatric symptoms. South Med J 1973:66:797-90.
3. Rosenthal MA. Carcinoid associated encephalitis successfully treated with tryptophan. J Clin Neurosci 2004;11:66-7.

4. Williams MD, Dolenic T. Selective serotonin reuptake inhibitors and patients with carcinoid tumor. Psychosomatics 2005:46:370-2.

\section{Oesophageal I}

\section{PTU-164 THE CLINICAL UTILITY OF THE ENDOSCOPIC FUNCTIONAL LUMINAL IMAGING PROBE IN EOSINOPHILIC OESOPHAGITIS: A CASE SERIES}

doi:10.1136/gutjnl-2012-302514c.164

${ }^{1}$ A Simpson, ${ }^{* 1} \mathrm{M}$ S J Wilson, ${ }^{2}$ A Ellefson, ${ }^{1}$ S Colley, ${ }^{1} \mathrm{~S}$ E Attwood. ${ }^{1}$ Department of Surgery, North Tyneside General Hospital, North Shields; ${ }^{2}$ Ardmore Healthcare Limited, Amersham, UK

Introduction Disease severity assessment in eosinophilic oesophagitis (EoE) is limited by the poor correlation of endoscopic appearance and histological eosinophil density with symptoms. Our aim is to ascertain whether having a measurement of oesophageal wall distensibility (using the EndoFLIP device) helps clinical decision making in the management of patients with EoE.

Methods Dysphagic patients with proven/suspected EoE were assessed by upper gastrointestinal endoscopy, oesophageal biopsies, clinical history, weight, and medication history. EndoFLIP assessment was carried out if symptoms persisted despite medical treatment or if there was an uncertain diagnosis. Assessment was performed under general anaesthetic. The $8 \mathrm{~cm}$ EndoFLIP balloon was inflated in the lower oesophagus, first to 20 , then 30 and up to $40 \mathrm{ml}$ of water according to oesophageal wall distensibility. After balloon emptying and repositioning in the upper oesophagus, the measurements were repeated. Oesophageal diameter was recorded at $5 \mathrm{~mm}$ intervals and distensibility calculated as changes in crosssectional area per $\mathrm{mm} \mathrm{Hg}$. If the lumen was poorly distensible and $<11 \mathrm{~mm}$ across, a through-the-scope balloon dilator was used to expand the areas depicted by EndoFLIP as being poorly compliant. Repeat EndoFLIP testing immediately after dilation revealed the improvement in distensibility. Patients were followed up at 3 months.

Results 6 patients in total were included, five of whom had biopsy proven EoE. In all six patients we found the EndoFLIP useful in guiding management. Two of the five patients with EoE were found to have poor oesophageal distensibility and underwent dilatation, following which a clear improvement in distensibility was seen. This correlated with an improvement in symptoms at follow-up. The remaining three patients with proven EoE had normal distensibility measurements and therefore did not undergo dilatation as a result of EndoFLIP testing. The single patient without EoE had normal distensibility measurements. Dilatation was undertaken following tertiary centre consultation on the basis of high-resolution manometry testing but symptoms returned after 3 months. There were no difficulties in performing the EndoFLIP test. There was a single complication of oesophageal mucosal tear following dilatation, which was of no clinical significance.

Conclusion Measuring the oesophageal wall distensibility may become a useful tool in the clinical assessment of EoE and may help to define the need for oesophageal dilatation and predict the outcome of such intervention.

Competing interests A Simpson: None declared, M Wilson: None declared, A Ellefson Employee of: Ardmore Healthcare Limited, S Colley: None declared, S Attwood: None declared. 